\title{
Yielding Behaviour of Martensite in Steel
}

\author{
Bevis HUTCHINSON, ${ }^{1) *}$ David LINDELL ${ }^{1)}$ and Matthew BARNETT ${ }^{2)}$ \\ 1) Swerea KIMAB Box 7047 SE-164 07 Kista Sweden. \\ 2) Institute for Frontier Materials Deakin University Geelong 3217 Australia.
}

(Received on September 16, 2014; accepted on January 29, 2015)

\begin{abstract}
Although martensite is recognised as a very strong phase in carbon steels, its initial yielding commences at low stresses and the tensile stress-strain curve shows a smooth, rounded form. Evidence is presented from x-ray diffraction to show that this behaviour is due to the presence of intra-granular stresses that are residues after the shear transformation from austenite to martensite. These internal stresses are reduced in magnitude by plastic deformation and also by tempering. Reduction of internal stress due to plasticity is shown by a decrease in XRD line broadening after deformation. A simple model is presented in which the stress-strain behaviour is controlled by relaxation of the internal stresses almost up to the point of the ultimate tensile strength. It demonstrates that only a very small fraction of the material remaining in a purely elastic state provides a large stabilising effect resisting necking. A corollary of this is that the uniform elongation of martensitic steel actually increases with increase in the strength level. Effects of heat treatment are also reproduced in the model, including the increase in conventional yield stress $\left(R p_{0.2}\right)$ that occurs after low temperature tempering.
\end{abstract}

KEY WORDS: steel; martensite; internal stress; plasticity; yielding.

\section{Introduction}

Martensite is well known to be a very strong phase in steels and has been studied extensively over many decades. In addition to its traditional uses such as in tools and springs, there is renewed interest in it for applications in vehicles because of the weight savings that can be achieved through down-gauging. Such applications, however, place demands not only on strength but also for some plasticity that is required during forming and for energy absorption in crash situations. This has stimulated considerable activity in recent years into new processing methods as well as into the microstructures of martensitic steels. Interest has centred, in particular, on the lath martensite structures found in lowmedium carbon steels. Current understanding and findings have been reviewed by Maki. ${ }^{1)}$ Following hardening by quenching it is common practice to temper the material at temperatures between $150^{\circ} \mathrm{C}$ and $700^{\circ} \mathrm{C}$ depending on the application. The present paper refers mainly to steel in the as-quenched (fresh martensite) condition although effects of tempering will also be included.

The tensile strength and conventional yield stress $(0.2 \%$ proof stress) are high compared to steels having other microstructures and they increase approximately linearly with carbon content. ${ }^{2)}$ The stress for initial plastic yielding is, however, very much less than this ${ }^{3-5)}$ and sometimes shows the opposite trend, with micro-yielding occurring at lower stresses as the carbon content is raised. ${ }^{6)}$ This phenomenon of initial yielding at low stress levels has been recognised

\footnotetext{
* Corresponding author: E-mail: bevis.hutchinson@swerea.se DOI: http://dx.doi.org/10.2355/isijinternational.55.1114
}

for more than 50 years and at least four different mechanisms have been invoked to account for it:

(i) Large densities of 'fresh' dislocations are created during the phase transformation and it has been argued that these can have high mobility, so eliminating yield point effects and causing a rounded stress-strain relationship at small loads. ${ }^{\text {e.g.7,8) }}$ Contrary to this view are results of Speich ${ }^{9)}$ and others, ${ }^{10}$ ) showing that virtually all the carbon is segregated to defects even immediately after quenching, so the dislocations should be rather fully locked by Cottrell atmospheres.

(ii) Retained austenite is normally present together with martensite and is a much softer phase. Krauss and co-workers ${ }^{2,5)}$ have argued that it is plasticity within the austenite that occurs at low strains and stresses. This viewpoint has some merit for high carbon steels where large contents of retained austenite occur but it seems unlikely in low carbon lath martensites where the fraction of austenite is often less than $1 \%{ }^{10}$ )

(iii) Recent atom probe results have shown that carbon is heterogeneously distributed in conventionally quenched martensitic steel. The structure may be regarded as a mixture of soft and hard 'elements' which yield successively at different stresses on loading, leading to a rounded stress-strain curve that commences at low stresses. ${ }^{11,12)}$ However, nano-hardness measurements on lath martensites show the hardness to be remarkably constant for a given material. ${ }^{13}$ ) Although there may exist variations of microstructure on an even finer scale than the nano-indents, such small scale elements could hardly be capable of 
independent mechanical behaviour.

(iv) Martensite always contains residual stresses after quenching and it has long been recognised that these may assist local yielding at low applied loads. In principle, both homogeneous long range (Type I) and inhomogeneous short range (Type II) internal stresses could be involved. The possibility that these can account for micro-yielding phenomena in martensite has been clearly demonstrated., ${ }^{3,414)}$ Type I stresses are typically associated with through-thickness gradients when the surfaces cool at faster rates than the centre whereas Type II stresses exist on a much finer scale of the effective grain size.

The present work builds on this last principle and concentrates on the role of Type II residual stresses since the materials are in the form of thin sheets where the Type I stresses are accordingly small. The novelty in this approach lies in demonstrating that the role of the internal stresses is much more persistent than previously has been supposed. In fact, such stresses play a dominant role in the stressstrain behaviour of martensite both before and beyond the conventional $0.2 \%$ proof yield stress and even up to the on-set of necking in tension. This view will be supported by experimental measurements and incorporated into a very simple model of yielding which is based on the criterion that crystals of martensite yield sequentially, during loading, when the combination of external stress and local residual stress reaches the plastic flow stress of the martensite.

\section{Experimental}

We concentrate here on the local Type II internal stresses that exist between different crystals in the metal, arising from the heterogeneous shearing that is intrinsic to the martensitic transformation. Such stresses can be assessed by $\mathrm{x}$-ray diffraction (XRD) of line broadening. There is typically more than one source of line broadening in XRD since dislocations present in the martensite also make a contribution This classical source of line broadening has been reviewed, for example by Warren ${ }^{15)}$ and is sometimes denoted as due to Type III stresses. The effect of dislocations is especially significant in the tails of the line profiles as these include extreme conditions of lattice distortion. Unlike these, Type II stresses are limited by the plastic flow stress of the metal so these are revealed in full width at half maximum (FWHM) measurements, as used here. Specimen surfaces were prepared by electropolishing and examined in a Brukers D8 diffractometer equipped with a Göbel mirror, using $\mathrm{CuK} \alpha$ radiation together with an energy dispersive detector to eliminate background intensity. Samples of the same steels were also furnace-cooled from $900^{\circ} \mathrm{C}$ to provide fine grained reference specimens of ferrite free from internal strains. For determination of line breadths (B), the $\mathrm{K} \alpha_{2}$ intensity was stripped away using the Rachinger method and the peaks were corrected for instrumental broadening $\left(\mathrm{B}_{\text {instr }}\right)$ assuming Gaussian behaviour, i.e.

$$
\mathrm{B}^{2}=\mathrm{B}_{\text {meas }}^{2}-\mathrm{B}_{\text {instr }^{2}}
$$

Experiments have been carried out on three steels with chemical compositions shown in Table 1. Steel B was hot rolled sheet while $\mathrm{F}$ and $\mathrm{G}$ were supplied after cold rolling and continuous annealing. All steels were heat-treated in a laboratory muffle furnace with austenitisation times of 10 minutes at $900^{\circ} \mathrm{C}$ followed by quenching into brine before testing. In some cases tempering was done in air-circulating furnaces. An MTS universal test machine with an optical extensometer was used for tensile testing at room temperature $\left(20 \pm 2^{\circ} \mathrm{C}\right)$. The parallel gauge lengths were $50 \mathrm{~mm}$ and the extensometer measured over the central $30 \mathrm{~mm}$ length. The strain rate was nominally $10^{-4} \mathrm{~s}^{-1}$.

\section{Initial Results}

Inserted in Fig. 1 are $2 \theta$ diffraction scans demonstrating (220) reflections for martensite and strain-free ferrite, normalised to the same height. The narrow ferrite peak corresponds to the instrumental broadening only and it is evident that martensite gives rise to a much wider spread of intensity. The full width at half maximum (FWHM, $\mathrm{B}_{2 \theta}$ ) breadths of the peaks were evaluated and then halved to give the broadening in terms of the Bragg angle, $\mathrm{B}_{\theta}$. Values of $\mathrm{B}_{\theta}$ for (110) and (220) peaks in the water-quenched martensitic steel B are plotted in the form of a Hall-Williamson graph in Fig. 1. Line broadening resulting from elastic strain should lead to a linear relationship that extrapolates to the origin in such a plot. It is evident that this is very nearly true and, accordingly, that the mean internal strain can then be derived as the slope of the line. Since there can be no resultant stress in such a structure, the positive and negative strains must be equal and can be evaluated as \pm 0.0044 in this case, which are quite large in the context of elasticity.

Data for four other steels having different carbon contents from a previous study ${ }^{10)}$ are reproduced here. Mean internal strains were derived from the $\mathrm{B}_{\theta}$ values of the (222) reflec-

Table 1. Chemical compositions of the investigated steels in wt.\%.

\begin{tabular}{ccccccc}
\hline STEEL & C & SI & MN & AL & TI & B \\
\hline B & 0.23 & 0.29 & 1.45 & 0.05 & 0.040 & 0.0034 \\
F & 0.20 & 0.21 & 0.98 & 0.04 & 0.024 & 0.0002 \\
G & 0.28 & 0.19 & 0.73 & 0.04 & 0.033 & 0.0019 \\
\hline
\end{tabular}

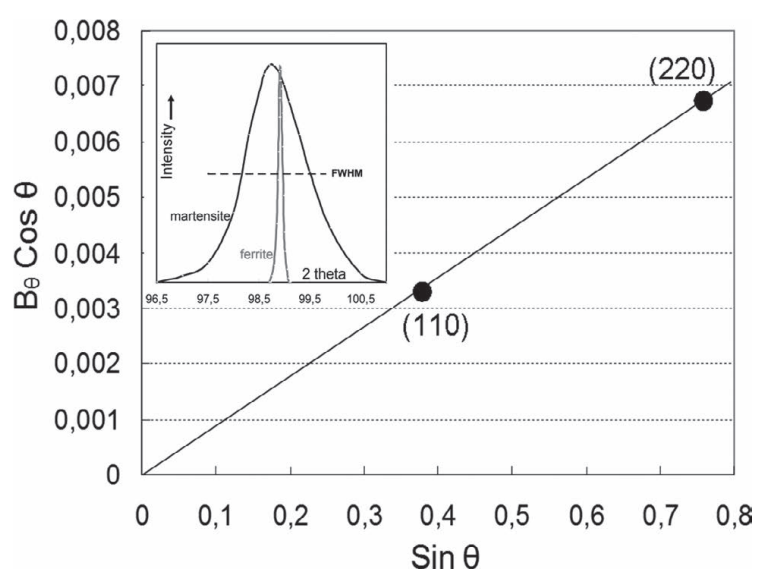

Fig. 1. Hall-Williamson plot for as-quenched steel B showing that line broadening arises almost entirely as a result of internal strains. Inserted (220) peaks for ferrite and martensite are scaled to the same height. 
tions, corrected for instrumental broadening. This reflection was chosen in case any tetragonality existed in the martensite since (222) is a unique spacing, independent of the c/a ratio (in fact, no tendency for tetragonality was detected in any of the steels). The strain values were transformed into equivalent stresses using a value of Young's modulus of 272 GPa which applies for the $<111>$ direction in iron normal to the diffraction planes, and these are plotted as the abscissa values in Fig. 2. Vickers hardness measurements on the four steels were converted into tensile strengths using the relationship $\mathrm{Rm}(\mathrm{MPa})=3.3 \mathrm{Hv}$ which is appropriate for steels of this strength level ${ }^{16)}$ and these are plotted on the ordinate of Fig. 2. It can be seen that the deduced internal stresses have values that are on average about half of the macroscopic strength for the four steels.

It may at first seem strange to invoke the tensile strength in a relationship of this nature, rather than, for example, the $0.2 \%$ proof stress. However, we consider that the tensile strength is actually the best available measure of the stress to cause plastic flow in the martensite as will be justified further below. For the purpose of clarity we point out that the internal stresses are not regarded as being the cause of strength in martensite. On the contrary, it is the intrinsic strength of the martensite that sets a limit to the magnitude of the internal stresses.

\section{Model for Yielding}

The observation that large internal stresses exist in the martensite and the realisation that these must influence the behaviour during loading provided the basis for a model. The principle adopted is similar to that proposed by Masing ${ }^{17)}$ who treated an aggregate of elements having different strengths, whereas here they have the same strength but different residual elastic stresses. The following assumptions are involved:

- Only residual shear stresses are present. Although this cannot be completely correct, it is a reasonable first approximation since the shape change during transformation is dominated by simple shear. We assume that elements in the microstructure have values of residual shear stress in a flat-top distribution ranging from zero

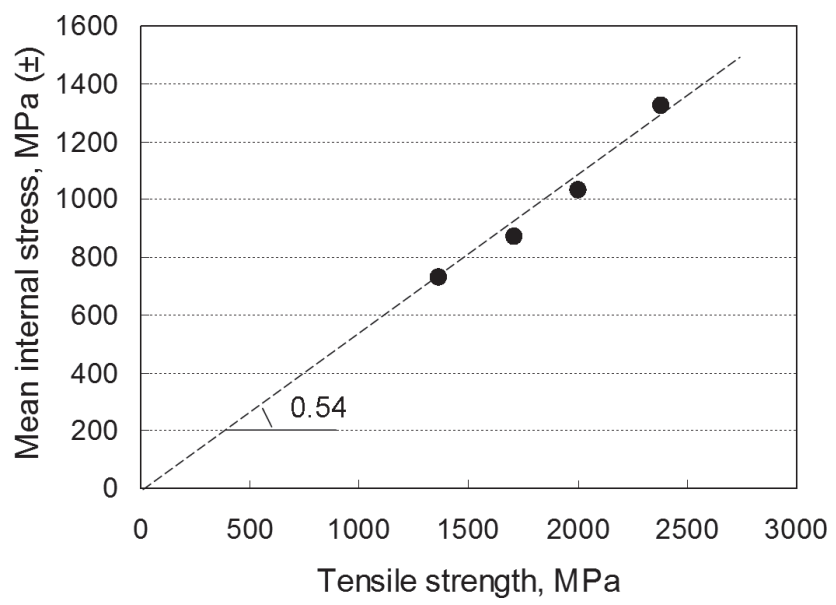

Fig. 2. Relationship between tensile strength and mean internal stress for four quenched martensitic steels with carbon contents in the range $0.12 \% \mathrm{C}$ to $0.48 \% \mathrm{C}$ from Ref. 10 ). up to a maximum. For fresh martensite the maximum is the same as its plastic flow stress since this had been plastically deformed during the transformation.

- Planes and directions of the local shear stresses are randomly distributed in space which should be a good approximation since texture and orientation effects are weak.

- Two stress magnitudes are defined. One is the maximum internal shear stress and the other is the critical stress to plastically deform the martensite (its flow stress). Tempering can modify one or both of these.

- True work hardening of the already dislocated martensite is expected to be small and has generally been set to zero here although a work hardening rate can be incorporated.

- Strains along the loading direction are assumed to be the same in all grains (iso-strain model). These may be purely elastic or elastic + plastic. This condition is unduly restrictive since the plastic elements are expected to flow more than the elastic ones, as discussed in e.g. ${ }^{11)}$ but it enables us to obtain a useful solution.

A schematic representation of the material as the basis for modelling is shown in Fig. $\mathbf{3}$ and more details are given in Appendix 1. Different microstructural elements in the metal have their internal stress axes distributed at random, described by $\Phi$, the angle between the normal $(\mathrm{N})$ to the shear stress plane and the tensile axis, and $\varphi_{1}$ which defines the direction of the shear within the plane. The calculations take into account the different fractions of material which vary as a sine function of the angle $\Phi$. As loading progresses in a tensile test, normal strains are imposed along the tensile direction, raising the value of $\sigma_{33}^{\prime}$ in the sample frame. The first elements to deform plastically are those having internal shear stresses near to $45^{\circ}$ in $\Phi$ and $90^{\circ}$ in $\varphi_{1}$. After each step, the elements are tested to see which ones have reached the Tresca yield condition.

A relevant question here concerns the size of the regions in the microstructure which constitute the internally stressed elements. At the present time there is no available method that can investigate this experimentally. We suppose that a relevant scale would be the block size of the martensite within which a common variant of transformation has taken

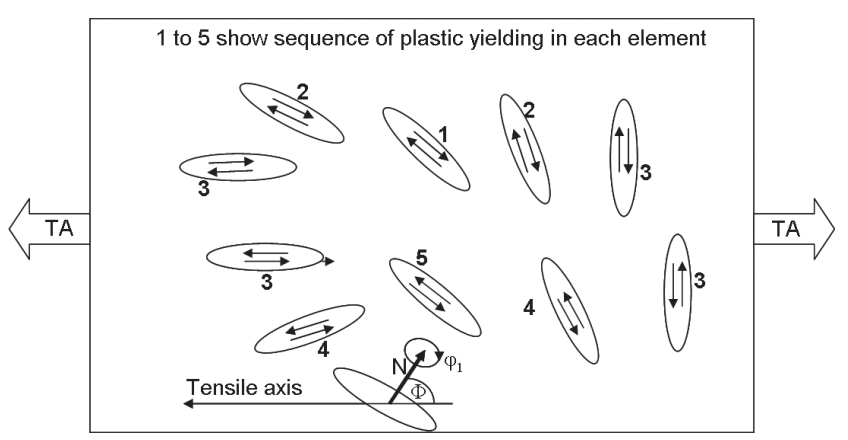

Fig. 3. Schematic diagram of elements in the microstructure of martensite having internal shear stresses and subjected to loading along the tensile axis TA. With increasing load the elements become plastic in the sequence 1 to 5 . Angle $\Phi$ $(0-\pi)$ defines the normal $(\mathrm{N})$ to the plane of the residual shear stress while $\varphi_{1}(0-2 \pi)$ defines its shear direction. 
place. Accordingly, the elements should be considerably smaller than the prior austenite grains.

After an element has started to yield, the normal stress along its tensile axis, $\sigma_{33}^{\prime}$, is not generally equal to the flow stress value, FS, since that element is still constrained by surrounding material. To describe the continuing deformation in a rigorous manner would necessitate a very sophisticated model which is hardly justifiable in view of the other conditions that are assumed here. We have adopted, therefore, a simplifying assumption whereby $\sigma_{33}^{\prime}$ in the element subsequently increases at a linear rate until the value FS is reached. This hardening rate originates in elastic relaxation and is expressed as a function of the elastic modulus, with a semi-empirical value that is derived from a comparison of calculated results and experimental stress-strain curves. Note that this does not refer to a conventional plastic work hardening condition caused by multiplication of dislocations. Other elements yield successively as the applied strain is increased until, finally, those having the least favourable internal stresses have yielded fully and, thereafter, the metal becomes completely plastic. The present approach uses an elementary iso-strain model. The possibility of alternatives such as the iso-work principle ${ }^{18)}$ was investigated but found not suitable for this purpose.

Figure 4 compares model calculations with experimental measurements on brine-quenched steel $G$ with the flow stress in the model being equated with the experimental true stress at instability. References to Young's modulus (E) for structural steels in the literature frequently cite a value of $210 \mathrm{GPa}$. However, this is not a true constant since it varies with crystallographic texture and is also somewhat lower in the case of martensite. ${ }^{19)}$ For the purpose of simplicity, it was taken to be $200 \mathrm{GPa}$. The assumed flat spectrum of residual shear stresses was approximated by equal volumes having values of $0, \pm 1 \mathrm{FS} / 8, \pm 2 \mathrm{FS} / 8, \pm 3 \mathrm{FS} / 8$ and $\pm 4 \mathrm{FS} / 8$. This maximum stress level was chosen on the assumption that the internal stresses are residues after the plastic deformation that accompanied the transformation from austenite to martensite on quenching and so can equal but not exceed the plastic flow stress, based on the Tresca criterion. The only adjustable parameter is the assumed hardening rate for $\sigma_{33}^{\prime}$ following the yield criterion. Closest agreement

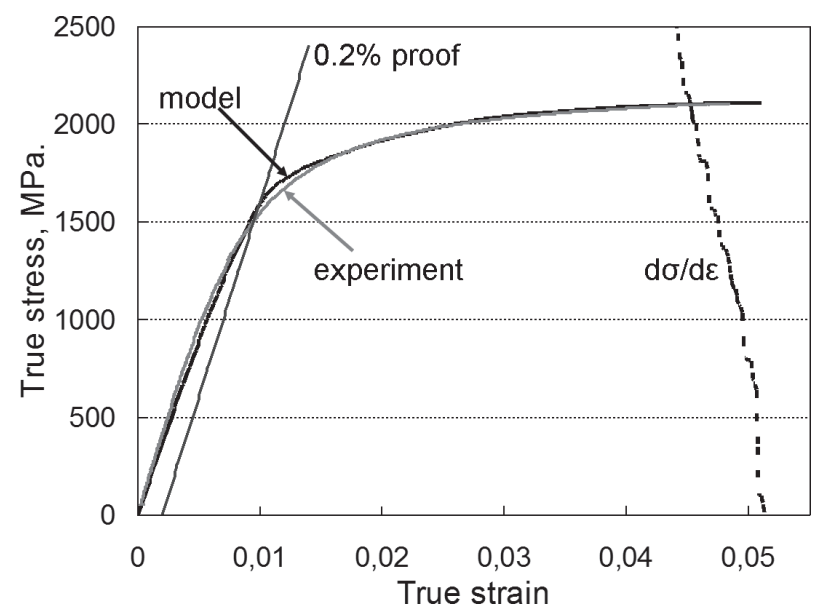

Fig. 4. Comparison of modelled and experimental stress-strain curves. The flow stress of $2100 \mathrm{MPa}$ in the model is matched to the true stress at the measured UTS. with experiment was found when this hardening rate was set as E/5 and that value has also been assumed in all other calculations.

The initial micro-yielding and rounded shape of the curve are captured by the model as well as some other features which will be discussed shortly. It may indeed be so that the good agreement in Fig. 4 is somewhat fortuitous in view of the assumptions involved. We do not view the model as being quantitatively precise in detail, nor is that its main aim. Rather, the purpose was to highlight certain features about yielding and flow in martensite and, from these, make predictions that can be tested with further experiment. A wide variety of different conditions were investigated including different residual stress patterns and hardening rates but, in fact, the qualitative inferences that we deduced were found to be independent of the actual values used as model parameters over a wide range.

Figure 5 shows an example of predicted stress-strain curves for as-quenched steels having assumed plastic flow stresses (FS) of $1000 \mathrm{MPa}$ and $1500 \mathrm{MPa}$. The very low elastic limit is evident as typically found in as-quenched martensites. In fact, in these cases, the first micro-yielding occurs already at the start of loading although the initial plastic deformation is so small as to be seemingly elastic in the diagram. The conventional $0.2 \%$ proof yield stresses are evaluated as $840 \mathrm{MPa}$ and $1200 \mathrm{MPa}$. Included in Figs. 4 and 5 are also the work hardening rates, $\mathrm{d} \sigma / \mathrm{d} \varepsilon$. Considère's criterion predicts that instability will occur where the two curves cross one another (circled) and that this will correspond to the UTS in a nominal stress-strain diagram. It is interesting to see that this instability occurs almost immediately before the steel becomes fully plastic. The reason is evidently that any remaining elements that are still elastic have a 'work hardening' rate, $\mathrm{d} \sigma / \mathrm{d} \varepsilon$, equal to Young's modulus which is extremely large and so is highly effective in resisting instability. This is, accordingly, the reason why the tensile strength rather than the $0.2 \%$ proof stress provides the best estimate of the plastic flow stress of martensite; only close to this point does the material become completely plastic.

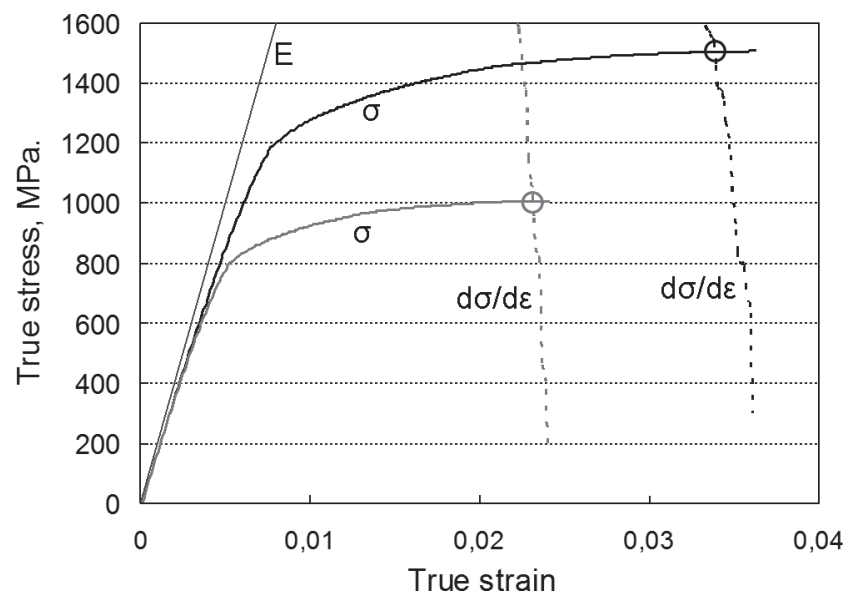

Fig. 5. Predicted stress-strain curves and work hardening rates, $\mathrm{d} \sigma / \mathrm{d} \varepsilon$, for martensitic steels having flow stresses of 1000 $\mathrm{MPa}$ and $1500 \mathrm{MPa}$ with the iso-strain model. 


\section{Predictions and Verification}

Despite the present model's recognised simplifications, it does allow some rationalisations to be made and also some surprising predictions that have been confirmed by experiment. Most significant of these predictions are:

- Smooth or gradual yielding behaviour should be normal in martensitic steels as discussed above.

- Relaxation of internal stresses by plastic flow should result in a reduction of the XRD line broadening after deformation, contrary to the normal behaviour of metals.

- Since instability under tensile loading is prevented so long as some microstructural elements remain in the purely elastic state, it predicts that increased strength of martensite will be accompanied by a greater uniform elongation. This may occur, for example, with higher carbon contents and is also contrary to usual expectations.

- Tempering at low temperatures may relax internal stresses without much reduction in the flow stress of the martensite. In such cases the conventional $0.2 \%$ yield stress should increase after tempering whilst the tensile strength is unaffected or even is reduced.

During yielding, the internal stresses are progressively relaxed as more and more elements reach the same flow stress value. Accordingly, the XRD line broadening is predicted to decrease after deformation. Two quenched samples that had been tensile strained to different degrees and one that was cold-rolled were analysed as described above and the results are presented in Figs. 6 and 7. The normal expectation that XRD line broadening should increase with deformation is not confirmed here. Instead, the lines become narrower, in conformity with the present hypothesis. Strain levels are reduced although not totally eliminated since the effect of dislocations is still present, as well as intra-granular stresses arising from anisotropy in different grains. The data in Fig. 7 are limited but they suggest that the internal stresses are minimised at around $2.5 \%$ plastic strain which corresponds to Considère instability and the UTS condition. This is where the model predicts the most complete elimination of the $\sigma_{33}^{\prime}$ stresses that were retained after phase transformation. Thereafter, the internal strains do rise with

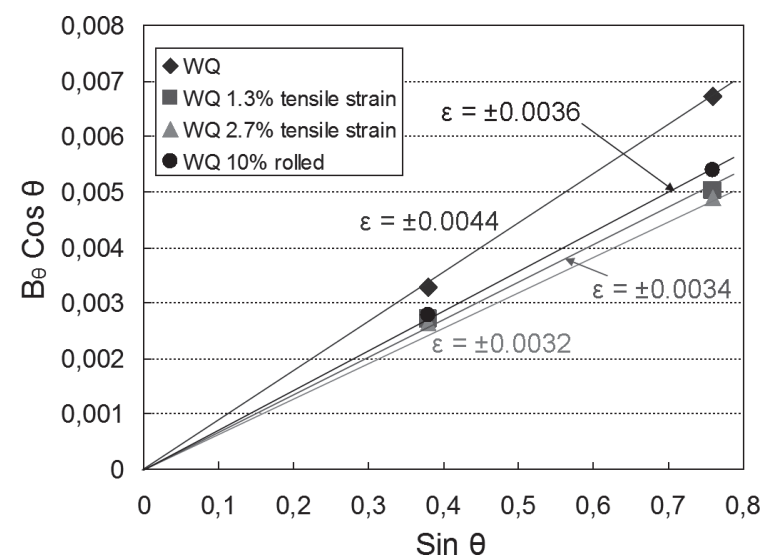

Fig. 6. Hall-Williamson plots for martensite in steel B after quenching and after subsequent plastic deformation. Symbol sizes indicate uncertainty in measurement on Y-axis. increasing plastic flow but only slowly and it appears as though large deformations would be necessary before these resume the level that existed in the virgin martensite.

The second prediction that uniform elongation should become greater as the strength of martensite increases can be seen in the circled locations in Fig. 5 and is exemplified by additional model calculations shown in Fig. 8. These apply to as-quenched conditions where the maximum internal stresses are assumed to be equal to the plastic flow stresses, for example in steels having a range of different carbon contents. Values for uniform elongation differ considerably depending on whether elastic contributions are included or omitted. Both situations may apply in practice, according to the way in which experimental measurements are carried out (whether the strains are measured during continuous loading or after unloading), so both are included in Fig. 8.

The prediction that the uniform elongation of martensitic steels increases with strength level was tested in tensile experiments on steels $\mathrm{F}$ and $\mathrm{G}$ carefully processed in an identical manner. Duplicate specimens cut out by spark machining were brine quenched after heating for 10 minutes at $900^{\circ} \mathrm{C}$. Some of these were tempered for 3 minutes at $200^{\circ} \mathrm{C}$ corresponding to a current industrial practice.

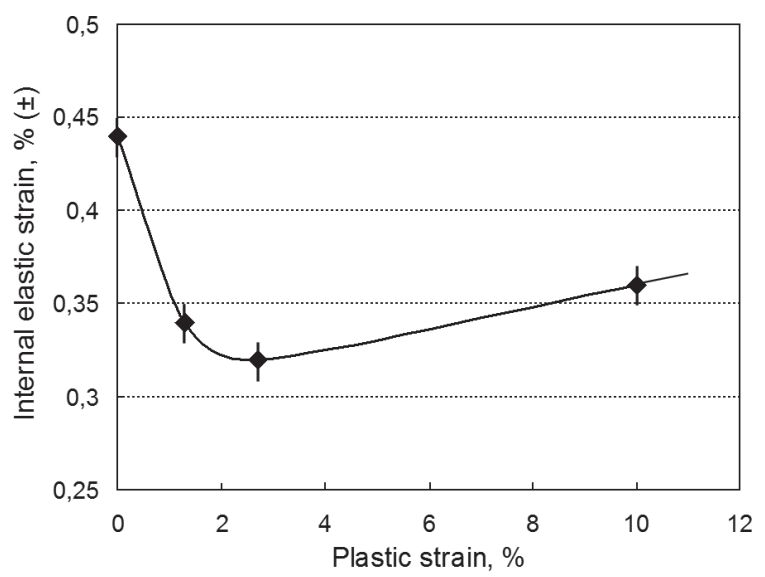

Fig. 7. Internal elastic strains $|\varepsilon / 2|$ calculated from XRD line breadths as a function of applied nominal plastic deformation.

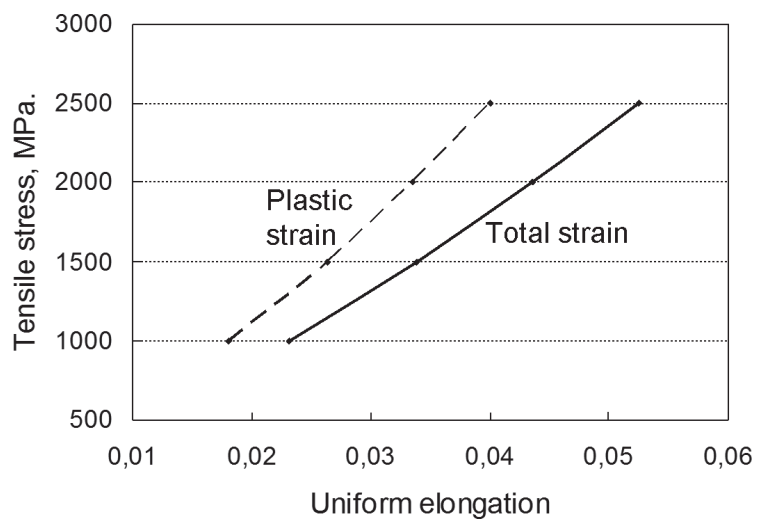

Fig. 8. Model calculations for as-quenched martensitic steels with different strength levels, showing the relationships between the strain to instability (uniform elongation) and tensile stress. The solid line shows elongation based on total strain while the dashed line is for plastic strain only. 
Figure 9 shows a plot of tensile stress versus uniform elongation, i.e. the strain to maximum load in the tensile test. It is evident that steel $\mathrm{G}$ with the higher strength level shows greater elongation than the softer steel F. Furthermore, tempering which reduces the tensile strength is accompanied by reduction in the uniform elongation in both cases. These tendencies are contrary to normal behaviour in metals, the so-called 'banana curve'. Higher strength naturally implies a greater contribution from elastic extension which is included in the data points shown with solid symbols in Fig. 9. However, when this contribution is subtracted (open symbols) a similar positive slope is retained, confirming that the plastic strain to instability does indeed increase with increasing strength level, in conformity with the model.

A further rationalisation that can be deduced from the modelling approach concerns another effect of tempering. Tempering can have the effects of softening the martensite but also of reducing the internal stresses present after quenching. At low temperatures the latter effect is expected to dominate while at higher temperatures both the internal stresses and the flow stress of the martensite will be reduced. Figure 10 records the reduction of internal strains measured by XRD line broadening after tempering steel $\mathrm{B}$ at two

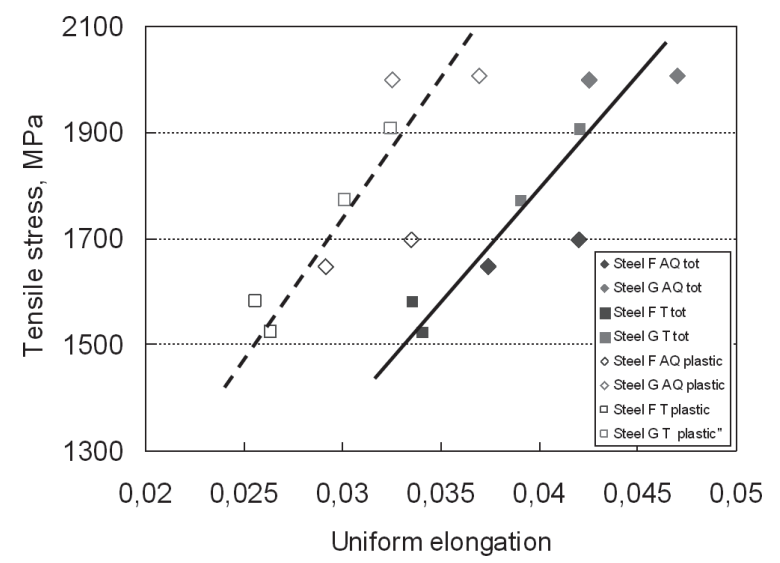

Fig. 9. Maximum tensile stress versus uniform elongation (strain) in tensile tests for steels F and G. AQ is as-quenched condition and $\mathrm{T}$ is tempered for 3 minutes at $200^{\circ} \mathrm{C}$. Solid symbols and line are for total strain. Open symbols and dashed line are for plastic strain only.

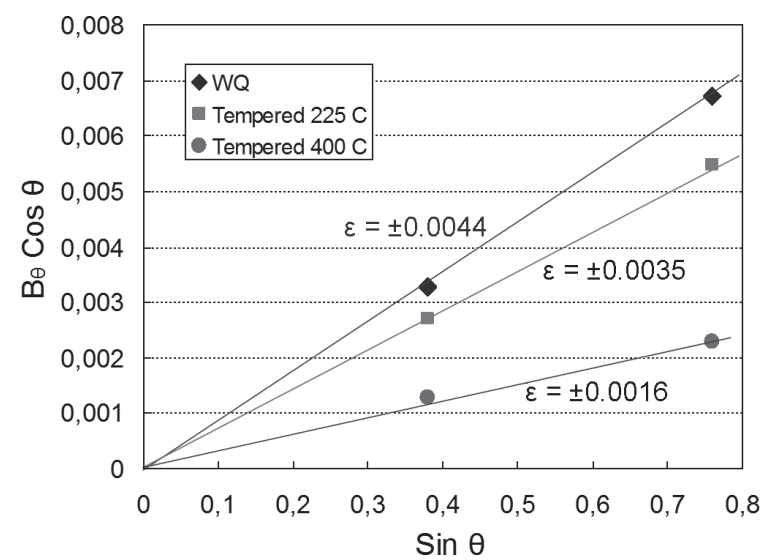

Fig. 10. Hall-Williamson plots demonstrating the reduction of internal strains after tempering steel B at two different temperatures. temperatures.

A simulation of low temperature tempering is shown in Fig. 11 where the calculations compare a curve for asquenched martensite with another where tempering has reduced all the internals stress levels by $20 \%$ without affecting the plastic flow stress which is set at $2100 \mathrm{MPa}$ in both cases. The highest residual shear stress in the flat distribution in the latter case is reduced to $\pm 840 \mathrm{Mpa}$. The tensile strength is unchanged but the conventional $0.2 \%$ proof yield stress increases by $280 \mathrm{MPa}$ and the elastic limit is also raised. Both these results are in good qualitative agreement with observations reported in the literature ${ }^{\text {e.g.3) }}$ and are supported by present measurements shown in Fig. 12. Although tempering at $200^{\circ} \mathrm{C}$ or $225^{\circ} \mathrm{C}$ somewhat reduced the tensile strength in all cases, it caused a significant increase in the yield stress as defined by the $R p_{0.2}$ values, due to the reduction of internal stresses. After the higher temperature treatment at $400^{\circ} \mathrm{C}$, the yield stress of steel B had fallen to almost the as-quenched value due to substantial reduction in the plastic flow stress, as evidenced by the much reduced tensile strength.

Figure 11 also demonstrates a decrease in the uniform elongation after tempering despite the flow stress and tensile strength remaining unchanged. This too was confirmed in experiments; for both steels $\mathrm{F}$ and $\mathrm{G}$ the uniform elongation reduced by 0.5 percentage units after tempering at $200^{\circ} \mathrm{C}$.

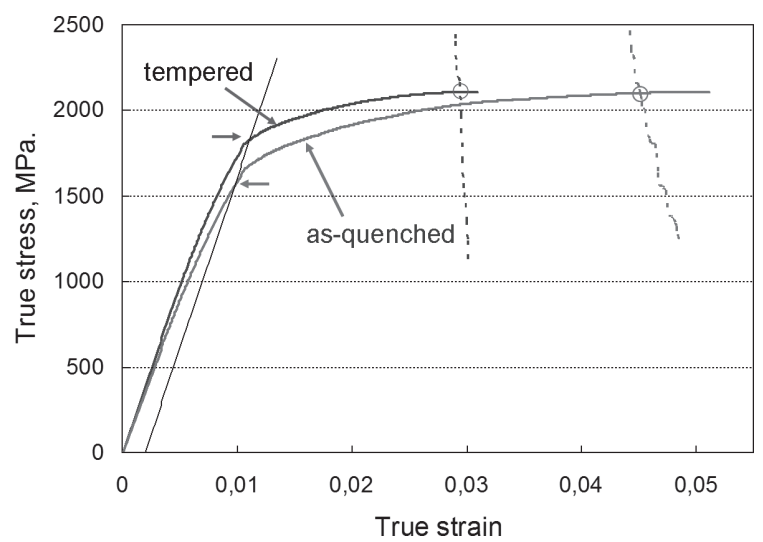

Fig. 11. Calculated stress-strain curves for fresh and tempered martensite. Tempering has reduced the internal stresses by $20 \%$ in this example without affecting the plastic flow stress.

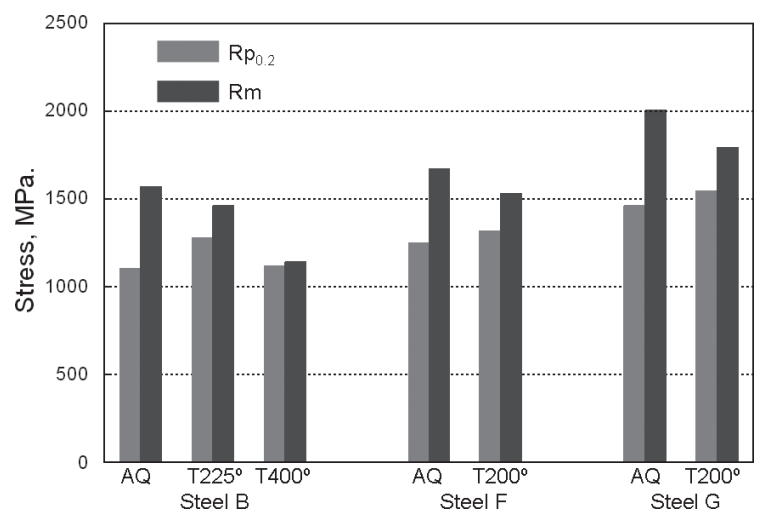

Fig. 12. Yield and tensile stresses for three steels in as-quenched (AQ) and tempered $\left(\mathrm{T}^{\circ} \mathrm{C}\right)$ conditions. 
The explanation for this is that plastic instability is deferred as long as some elements in the structure remain in an elastic state. The higher are the initial compressive stresses along the testing direction, the greater must be the applied strain before these elements yield. Relaxation of the internal stresses therefore leads to earlier instability and reduced uniform elongation.

\section{General Discussion}

The possibility that residual stresses from the transformation may play a role in the initial yielding of martensite has been recognised for many years ${ }^{3,4,14}$ although several other explanations have also been suggested. Our present approach attempts to make this more quantitative and to demonstrate that the effect is much more persistent than was previously believed. The model, although elementary, supports the viewpoint that purely elastic elements in the microstructure can persist during tensile testing up to virtually the ultimate tensile stress. In simple terms, some elements in the initial material exist with very high effective tensile stresses that may actually equal the plastic flow stress along the direction of loading. Only a very small load needs to be applied for these regions to yield which accounts for the low micro-yield points that have been reported in fresh martensite. ${ }^{4,5)}$ Other regions that are less favoured elastically require greater applied strains before they become plastic. The most extreme situation is found in elements of the microstructure that were initially compressed since these must be first stretched into tension and then beyond, until the condition for plasticity is reached.

The present work ignores any true work hardening in the plastically deforming elements although that can easily be incorporated into the model. The dislocation densities remaining in martensite after its transformation from austenite are of the order of $10^{15} \mathrm{~m}^{-2}$ from TEM measurements ${ }^{20)}$ and so resemble those in heavily cold worked metals. These structures ought to correspond to linear Stage IV behaviour which has a very low value of work hardening and, indeed, this is true of martensite at higher strain levels. Zaccone and $\mathrm{Krauss}^{5)}$ commented on the very high work hardening rate in martensite but, in fact, this only applies in the early stages of yielding. The present analysis leads us to concur with Leslie and Sober") in their conclusion that 'the apparent initial rate of work hardening is only a reflection of the rapid elimination of internal stress concentrations', that is to say that it is determined by the continuing extended elasto-plastic transition.

An important question to address is whether the internal stresses are, indeed, as large as is assumed here. Two arguments can be put forward to support this supposition. The mean internal stresses determined from the broadening of XRD lines correspond to about half of the plastic flow stress and the broad diffraction lines imply that some regions contain much larger strains than these. Line broadening is most commonly attributed to dislocations and then the precise nature of the overall strain fields will depend on the arrangements of the dislocation networks. In some cases, such as dipole arrangements, the dislocation contribution to longer range stresses is small. In others where dislocations exist in pile-ups, they will accommodate large regions of high elas- tic strain due to their combined stress fields. Bearing in mind that the formation of martensitic involves large strains in individual grains as the transformation shears of magnitude $\sim 0.2$ are resisted by neighbouring structures, it is reasonable to suppose that dislocation structures are polarised in such a manner. While the interpretation of line broadening due to dislocations is well established ${ }^{15)}$ it should also be noted that Type II intra-granular stresses may exist without the presence of any dislocations at all. Conceptually, grains could be strained into different elastic states and then joined together in an equilibrated arrangement where some of the strains are retained. Fonseca et $a .^{21)}$ pointed out that both elastoplastic self consistent (EPSC) models and crystal plasticity finite element models (CPFEM) predict the development of Type II intra-granular elastic strains during deformation. However, neither of these approaches invokes the existence of dislocations so the Type II strains must provide a source of line broadening that is separate from and superimposed upon that due to dislocations. In their work on tensile deformation of IF steel, the dislocation contribution was larger than that of the Type II stresses predicted by CPFEM. Present results indicate that the contrary is true in the case of martensite. This is reasonable since the internal stress or strain level (plastic flow condition) for martensite is at least six times greater than for the strained IF steel ${ }^{21)}$ while the $\mathrm{x}$-ray scattering from a dislocation should be similar in both.

The second argument is based on mechanics. Shearing during phase transformation is associated with plastic deformation of the martensite and, accordingly, the local stresses at that time must be equal to its plastic flow stress. Since there is no possibility of mechanical un-loading (as in a tensile test) these stresses must be remain locked-in, while the low martensite transformation temperature in combination with the rapid cooling means that very little thermal relaxation can be expected thereafter. Residual internal stresses up to the magnitude of the plastic flow stress are therefore to be expected. Indeed, phase field modelling of the martensite transformation by Malik et al. ${ }^{22)}$ has confirmed that regions in the microstructure should exist with residual stresses equalling the von Mises plastic flow stress of the martensite.

The present experiments involving XRD after plastic straining were carried out to test the predictions of the model that line broadening should become smaller after deformation, and this was found to be true. We discovered that the same phenomenon had already been reported by Takaki et al. ${ }^{23)}$ who interpreted it in terms of dislocations, using a simplified method to calculate dislocation densities from the XRD line breadths and implying a reduction of dislocation density by more than $50 \%$ due to straining. Since the deduced dislocation density in their quenched (low carbon 18\% nickel) steel was much greater than in a heavily cold rolled condition of the same strength, they reached the unusual conclusion that not all the dislocations in the martensite contribute to strengthening. A related paper ${ }^{7)}$ claimed a reduction of dislocation density by a factor of two based on TEM micrographs after a strain of only $0.02 \%$. However, no statistics of dislocation density were reported and there are great difficulties in drawing quantitative conclusions from TEM images. We consider that the present interpretation is more consistent in that it does not necessitate any reduction of dislocation density on straining. 
The XRD line breadths become smaller due to the reduction in intra-granular stresses and strains which are progressively relaxed as plasticity takes place.

Neutron diffraction experiments have been carried out insitu by Morooka et al. ${ }^{24)}$ who measured the line profiles with increasing tensile loads. These authors also reported that the peaks became narrower during deformation in the case of as-quenched martensite. Examination of their line profiles shows that the high d-spacing side of the peaks, corresponding to tensile strain, remained almost unchanged while the compressive, low d-spacing, side moved to higher values, thereby reducing the width of the peaks. This is precisely the situation that corresponds to our present interpretation and model. The plastic elements reach a constant tensile elastic strain while the elements that were initially in compression move progressively towards the same tensile elastic limit. These results support rather convincingly the view that the deformation of martensite should be considered as a process in which different volumes of the material undergo plastic yielding at different times, dependent on the stress states that reside inside them following their transformation from austenite. The present authors have confirmed the behaviour reported by Morooka et al. ${ }^{24)}$ during in-situ loading of steels $\mathrm{F}$ and $\mathrm{G}$ in synchrotron XRD experiments. The asymmetrical sharpening of the diffraction peaks during deformation in both neutron and X-ray diffraction is fully in agreement with the present model based on Type II stresses but is not consistent with a simple reduction in the dislocation density.

The present model for yielding in martensite is not to be considered as strictly correct; it involves simplifications, such as the iso-strain condition which is probably overrestrictive, but it provides understanding and also allows qualitative predictions to be made that are confirmed in experiments. For example, this model demonstrates the principle that a very small fraction of residual elastic elements has a profound stabilising influence, resisting the on-set of necking in tension. The stronger is the steel, the greater is the internal stress level and, consequently, the greater must be the applied strain before the last elements reach the yielding condition. As a result, the elongation to maximum load in a tensile test is predicted to become greater as the strength of martensite increases, in agreement with experiments (Fig. 9). Similar behaviour can also be seen, although not commented, in published results ${ }^{6}$ ) for lightly tempered steels with carbon contents in the range 0.3 to $0.5 \%$.

Additionally, the model provides a basis for understanding the changes that take place during tempering, where both increases and decreases in the conventional yield stress may occur, depending on the respective degrees of stress relaxation and recovery. Other workers have also suggested that reduction of internal strains can account for an increase in yield stress after tempering at low temperatures ${ }^{3,4)}$ although the present work is the first time that this has been put on to any quantitative basis so far as we are aware. Other changes also take place in the steel during tempering, leading to the formation of carbide particles, and these will also affect the mechanical behaviour. Nevertheless, it is known that the dislocations in lath martensites are very heavily loaded with carbon already in the as-quenched condition ${ }^{9,10)}$ so that further Cottrell locking of dislocations is unlikely to play a significant role in yielding after tempering.
Residual stresses in the martensite remaining after the phase transformation on cooling must be more varied and complex than the states of pure shear which were assumed here. For this reason alone, the model results cannot be expected to mimic experimental ones perfectly. Observations made during in-situ tensile tests of martensite in the scanning electron microscope ${ }^{25)}$ showed, among other things, localised shearing at some packet boundaries and prior austenite grain boundaries. The transformation strains associated with different grains and packets will generally occur in different senses and directions such that residual inter-granular shear stresses will frequently be locked in along their boundaries. Some of these regions, where the internal stresses are aligned with the external load, will favour early yielding on loading whereas others will resist it. These observations need not, therefore, be taken as evidence that the boundary regions are less strong than the rest of the material

\section{Conclusions}

Internal strains which are residues after the shear transformation from austenite play a major role in the yielding of martensite under applied loads. Line broadening in x-ray diffraction experiments shows that these strain levels are compatible with internal stresses that may equal the plastic flow stress. Micro-yielding during tensile tests occurs at low applied loads due to plasticity in regions of the structure where there are high positive internal stresses. However, the presence of large negative internal stresses in combination with the high strength of martensite mean that tensile elongations typically $>2 \%$ must be applied before the whole material becomes plastic. Instability in tension is strongly resisted by material elements that are still elastic so that the Considère criterion corresponds closely with the point where the whole material becomes plastic. Accordingly, it is the ultimate tensile stress which provides the best measure of the true flow stress of the martensite.

A simple model constructed on these principles demonstrates many relevant features of yielding and flow up to the UTS. The expectation that internal stresses should reduce with applied deformation have been confirmed by the reduction of XRD line widths, in agreement with observations made by others. Furthermore, the model approach predicts that the uniform elongation in tensile tests should increase with increasing strength for martensitic steels and this has also been confirmed. Effects of tempering on tensile behaviour of the martensite can also be rationalised with the aid of the model. The conventional $(0.2 \%$ proof) yield stress is raised due to relaxation of internal stresses after low tempering temperatures but it decreases after higher temperature treatment due to softening of the martensite. Measurements made after tempering are in conformity with qualitative predictions of the model.

\section{Acknowledgements}

The author thanks Dan Jacobsson at SwereaKIMAB for carrying out the $\mathrm{X}$-ray diffraction measurements. They also thank John Duncan for pointing out an error in an earlier version of the model and Lena Ryde who has been a valuable partner in discussions. This work has been supported 
and partly financed by SSAB-EMEA with the encouragement of Ylva Granbom and materials supplied by Anders Haglund. BH expresses his thanks to Deakin University in Australia for provision of facilities during part of the work and preparation of the manuscript.

\section{REFERENCES}

1) T. Maki: Proc. 1st Int. Symp. on Steel Sciences, ISIJ, Tokyo, (2007), 1.

2) G. Krauss: ISIJ Int., 35 (1995), 349.

3) H. Muir, B. Averbach and M. Cohen: Trans ASM, 47 (1955), 380.

4) W. Leslie and R. Sober: Trans. ASM, 60 (1967), 459

5) M. Zaccone and G. Krauss: Metall. Mater.Trans., 7 (1989), 188.

6) M. Saeglitz and G. Krauss: Metall. Mater. Trans., 28 (1997), 377.

7) A. J. McEvily, R. C. Ku and T. L. Johnson: Trans. AIME, 236 (1966), 108.

8) K. Nakashima, Y. Fujimura, H. Matsubayashi, T.Tsuchiyama and S. Takaki: Proc. 1st Int. Symp. on Steel Sciences, ISIJ, Tokyo, (2007), 215.

9) G. Speich: Trans. AIME, 245 (1969), 2553.

10) B. Hutchinson, J. Hagström, O. Karlsson, D. Lindell, M. Tornberg, F. Lindberg and M. Thuvander: Acta Mater., 59 (2011), 5845.

11) S. Allain, O. Bouaziz and M. Takahashi: ISIJ Int. 52 (2012), 717.

12) G. Badinier: PhD thesis, University of British Columbia, (2013).

13) L. Zhang, T. Ohmura and K. Tsuzaki: Nanoindentation in Materials Science, chapter 5, INTECH, Croatia, (2012).

14) C. Magee and H. Paxton: Trans. AIME, 242 (1968), 1766.

15) G. Masing: Wissenschaftliche Veroffentlichungen aus dem SiemensKonzern, 3 (1923), 231.

16) Engineering Handbook.com, (2004-2006), (accessed 2013).

17) F. Montheillet and G. Damamme: Adv. Eng. Mater., 7 (2005), 852.

18) S. Morito, J. Nishikawa and T. Maki: ISIJ Int., 43 (2003), 1475.

19) B. Reitinger, T. Berber, O. Helm and P. Burgholzer: Proc. 1st Int. Symp. on Laser Ultrasonics, NDT.net, Germany, (2008), Paper 33.

20) B. E. Warren: Prog. Met. Phys., 8 (1959), 147.

21) J. Q. da Fonseca, E. C. Oliver, P. S. Bate and P. J. Withers: Mater. Sci. Eng., A437 (2006), 26.

22) A. Malik, H. K. Yeduu, G. Amberg, A. Borgenstam and J. Ågren: Mater. Sci. Eng., A556 (2012), 221.

23) S. Takaki, Y. Fujimura, K. Nakashima and T. Tsuchiyama: Mater. Sci. Forum, 539-543 (2007), 228.

24) S. Morooka, P. G. Xu, T. Suzuki, Y. Tomata and T. Kamiyama: Proc. 1st Int. Symp. on Steel Sciences, ISIJ, Tokyo, (2007), 207.

25) L. Ryde, D. Lindell and Y. Granbom: Dynamic SEM/EBSD Study of Martensite Deformation, SwereaKIMAB, Sweden, to be published.

\section{Appendix. 1 Principles in the model calculations}

The initial structure is assumed to exist in elastic states of shear described by stress tensors $\sigma$ with respect to the local shear axes. Values of $\sigma$ form a flat distribution from $-\mathrm{FS} / 2$ to $+\mathrm{FS} / 2$ where $\mathrm{FS}$ is the conventional plastic flow stress in tension.

$$
\sigma=\left[\begin{array}{lll}
0 & 0 & \sigma \\
0 & 0 & 0 \\
\sigma & 0 & 0
\end{array}\right]
$$

Material elements are defined by the angle $\Phi$ between their shear plane normal direction $(\mathrm{N})$ and the applied stress axis (TA) from $0^{\circ}$ to $180^{\circ}$ together with the shear direction defined by the angle $\varphi_{1}$ in the range from 0 to $360^{\circ}$; see Fig. 3 . The contributions from different elements in the structure are weighted according to sine $\Phi$ to take into account their frequency in a random spatial distribution. The angles $\Phi$ and $\varphi_{1}$ were stepped in intervals of 3 degrees in the calculations The rotation matrix that transforms the local stress into the specimen coordinates is $\boldsymbol{R}$, where:

$$
\boldsymbol{R}=\left(\begin{array}{ccc}
\cos \varphi_{1} & \sin \varphi_{1} & 0 \\
-\cos \Phi \sin \varphi_{1} & \cos \Phi \cos \varphi_{1} & \sin \Phi \\
\sin \Phi \sin \varphi_{1} & -\sin \Phi \cos \varphi_{1} & \cos \Phi
\end{array}\right)
$$

The stress tensor in specimen coordinates is then $\sigma^{\prime}$ where:

$$
\sigma^{\prime}=\boldsymbol{R} \sigma \boldsymbol{R}^{\mathrm{T}}
$$

Tensile strain is applied in increments of $10^{-4}$ along the axis 3 in each loading step which increases the stress tensor element $\sigma_{33}^{\prime}$ by an amount $10^{-4} \mathrm{E}$ where E is Young's modulus. The principle stresses are then obtained as the eigenvalues $\lambda_{1}>\lambda_{2}>\lambda_{3}$ so that the Tresca condition for yielding in each material element can be tested as:

$$
\lambda_{1}-\lambda_{3}>\mathrm{FS}
$$

After each loading step, the average stress is found by summation over $\sigma_{33}^{\prime}\left(\Phi, \varphi_{1}\right) \sin \Phi$ and normalising. After each material element has reached the yield criterion, its value $\sigma_{33}^{\prime}$ is raised in successive loading steps with a chosen hardening rate (E/5) until this attains the limiting value (FS). Calculations were done using the software package Matlab. 